Original Article

\title{
Comparison of anterior gluteus medius fiber activation during general exercises and PNF exercises
}

\author{
SUNG-KWANG JU' ${ }^{1)}$, WON-GYU YoO²* \\ 1) Department of Physical Therapy, The Graduate School, Inje University, Republic of Korea \\ 2) Department of Physical Therapy, College of Biomedical Science and Engineering, Inje University: \\ 607 Obangdong, Gimhae, Gyeongsangnam-do 621-749, Republic of Korea
}

\begin{abstract}
Purpose] This study compared the activation of anterior gluteus medius fibers during general exercises and proprioceptive neuromuscular facilitation exercises. [Subjects and Methods] The study enrolled 15 healthy adults. The participants performed general hip abductor strengthening exercises and proprioceptive neuromuscular facilitation exercises; during both types of exercise, electromyography activity was recorded. [Results] Greater anterior gluteus medius fiber activation was observed during the proprioceptive neuromuscular facilitation exercises compared with the general hip abductor strengthening exercises. The anterior gluteus medius fibers exhibited greater activity during pattern 2 exercises compared with any other type of exercise. [Conclusion] The results suggest that pattern 2 exercises can selectively activate anterior gluteus medius fibers.

Key words: Gluteus medius, Strengthening exercise, PNF
\end{abstract}

(This article was submitted Aug. 10, 2016, and was accepted Dec. 1, 2016)

\section{INTRODUCTION}

The gluteus medius (GM) muscle is a primary hip abductor that provides pelvic stability during walking and other activities ${ }^{1)}$. O'Sullivan et al. suggested that rehabilitation protocols, comprising various weight-bearing exercises, should be varied to activate different GM regions ${ }^{2)}$. The anterior GM fibers are maximally active during the stance and single support phases ${ }^{3)}$. Several therapeutic exercise protocols focus on GM activity for rehabilitation and injury prevention in clinical and athletic training settings ${ }^{1)}$. Distefano et al. reported that such exercises are useful for patients who may not be able to perform weightbearing exercises, such as the wall-press and pelvic drop, which also activate the $\mathrm{GM}^{4}$ ). Proprioceptive neuromuscular facilitation (PNF) exercises involve the stretching of muscles through rhythmic joint movements and manual resistance, to enhance neuromuscular responsiveness by stimulating proprioceptors ${ }^{5)}$. PNF exercises may enhance performance more effectively than conventional single-plane or single-direction weight-training programs. Therefore, we compared the activation of the anterior GM fibers while performing wall-press, pelvic drop, and PNF exercises.

\section{SUBJECTS AND METHODS}

The study enrolled 15 healthy males (mean age $=29.1 \pm 2.9$ years; mean height $=173.4 \pm 7.1 \mathrm{~cm}$; mean weight $=71.7 \pm$ $8.5 \mathrm{~kg}$ ) with no history of musculoskeletal or neurological disorders. The study purpose and methods were explained to the subjects, who provided informed consent according to the principles of the Declaration of Helsinki prior to participation. Electromyography (EMG) activity was recorded using a surface MP150 (BIOPAC Systems, Santa Barbara, CA, USA). We measured the anterior GM fibers in the region equidistant from the anterior superior iliac spine and greater trochanter. The

*Corresponding author. Won-gyu Yoo (E-mail: won7y@inje.ac.kr)

(C2017 The Society of Physical Therapy Science. Published by IPEC Inc.

This is an open-access article distributed under the terms of the Creative Commons Attribution Non-Commercial No Derivatives (by-nc-nd) License $<$ http://creativecommons.org/licenses/by-nc-nd/4.0/>. 
EMG data were normalized to the maximum voluntary isometric contraction (MVIC), which represents the standard manual muscle test position for GM normalization. Participants performed two general hip abductor strengthening exercises (wallpress and pelvic drop exercises) $)^{2}$, and two PNF exercises (pattern 1, lower extremity flexion - abduction - internal rotation pattern; and pattern 2, lower extremity extension - abduction - internal rotation pattern). Muscle activation was recorded for $5 \mathrm{~s}$ during each exercise. The data were analyzed using SPSS for Windows software (ver. 20.0; SPSS Inc., Chicago, IL, USA). Repeated-measures one-way analysis of variance (ANOVA) was used to assess differences in the activity of the anterior of GM fibers during the exercises, with the significance level set at $\alpha=0.05$.

\section{RESULTS}

Greater muscle activity was observed during the PNF exercises compared with the general hip abductor strengthening exercises $(\mathrm{p}<0.05)$. The anterior GM fibers were activated more during PNF $2(63.58 \% \mathrm{MVIC}, \mathrm{p}<0.05)$ than during any other type of exercise (wall press: 17.41\% MVIC; pelvic drop: $25.40 \%$ MVIC, PNF 1: 52.34\% MVIC).

\section{DISCUSSION}

The GM was significantly more active during the two PNF exercises compared with the general hip abductor strengthening exercises. PNF engages the sagittal, frontal, and transverse planes of movement, with increased muscle activity extending distally and proximally6). Cross-training can enhance strength on the weaker side of the body during PNF. A recent study applied PNF (leg pattern) to one leg and the strength in the other leg increased significantly ${ }^{6}$. The anterior GM fibers were significantly more active during PNF 2 than PNF 1. Pattern 2 PNF is characterized by an extension-abduction-internal rotation pattern, while a flexion-abduction-internal rotation movement pattern is observed in PNF 1. The anterior GM fibers are activated during hip abduction, internal rotation, and assisted flexion ${ }^{1-3)}$. Earl demonstrated increased anterior GM fiber activity during an alternative weight-bearing exercise that requires internal hip rotation to maintain pelvic and hip stability 3,4 . The extremity flexor and extensor slings are activated during reciprocal gait $\left.{ }^{6}\right)$. Throughout the gait cycle, these two chains act alternately in a facilitative and inhibitory capacity with respect to the reciprocal left and right limb activity. When both slings are activated simultaneously, the lower extremities are stabilized. Future studies should examine GM activation that includes another sub-region in subjects with musculoskeletal dysfunction. In conclusion, greater GM muscle activation occurs during PNF compared with general hip abductor strengthening exercises. Moreover, pattern 2 PNF exercises selectively improve anterior GM fiber activation.

\section{REFERENCES}

1) Fredericson M, Cookingham CL, Chaudhari AM, et al.: Hip abductor weakness in distance runners with iliotibial band syndrome. Clin J Sport Med, 2000, 10: 169-175. [Medline] [CrossRef]

2) O'Sullivan K, Smith SM, Sainsbury D: Electromyographic analysis of the three subdivisions of gluteus medius during weight-bearing exercises. Sports Med Arthrosc Rehabil Ther Technol, 2010, 2: 17. [Medline]

3) Soderberg GL, Dostal WF: Electromyographic study of three parts of the gluteus medius muscle during functional activities. Phys Ther, 1978, 58: 691-696. [Medline]

4) Distefano LJ, Blackburn JT, Marshall SW, et al.: Gluteal muscle activation during common therapeutic exercises. J Orthop Sports Phys Ther, 2009, 39: 532-540. [Medline] [CrossRef]

5) Kofotolis N, Vrabas IS, Vamvakoudis E, et al.: Proprioceptive neuromuscular facilitation training induced alterations in muscle fibre type and cross sectional area. Br J Sports Med, 2005, 39: e11. [Medline] [CrossRef]

6) Kofotolis ND, Kellis E: Cross-training effects of a proprioceptive neuromuscular facilitation exercise programme on knee musculature. Phys Ther Sport, 2007, 8: 109-116. [CrossRef] 